\title{
Clopidogrel Bioactivation and Risk of Bleeding in Patients Cotreated With Angiotensin-Converting Enzyme Inhibitors After Myocardial Infarction: A Proof-of-Concept Study
}

\author{
KE Kristensen ${ }^{1}$, H-J Zhu ${ }^{2}$, X Wang ${ }^{2}$, GH Gislason ${ }^{1,3}$, C Torp-Pedersen ${ }^{4}$, HB Rasmussen ${ }^{5}$, JS Markowitz ${ }^{6}$ \\ and PR Hansen ${ }^{1}$
}

Clopidogrel is an oral antiplatelet prodrug, the majority of which is hydrolyzed to an inactive metabolite by hepatic carboxylesterase 1 (CES1). Most angiotensin-converting enzyme inhibitors (ACEls) are also metabolized by this enzyme. We examined the effects of ACEls on clopidogrel bioactivation in vitro and linked the results with a pharmacoepidemiological study. In vitro, ACEls inhibited CES1-mediated hydrolysis of a model substrate, and trandolapril and enalapril increased formation of clopidogrel active metabolite. In 70,934 patients with myocardial infarction, hazard ratios for clinically significant bleeding in ACEl-treated patients cotreated with or without clopidogrel were 1.10 (95\% confidence interval ( $\mathrm{Cl}): 0.97-1.25, P=0.124)$ and 0.90 (95\% $\mathrm{Cl}: 0.81-0.99, P=0.025)$, respectively, as compared with patients who did not receive ACEls. This difference was statistically significant $(P=0.002)$. We conclude that cotreatment with selected ACEls and clopidogrel may increase the risk of bleeding. Combination of in vitro and pharmacoepidemiological studies may be a useful paradigm for assessment of drug-drug interactions.

Platelet inhibition by clopidogrel plays an important role in the prevention of thromboembolic events after myocardial infarction (MI) and percutaneous coronary intervention (PCI). ${ }^{1,2}$ However, treatment with clopidogrel is associated with a significant risk of bleeding, which has been partially attributed to genetic predisposition, drug interactions, and other factors. ${ }^{3}$ Clopidogrel is a prodrug that, after absorption, is extensively metabolized by two major hepatic enzyme systems, carboxylesterase 1 (CES1) and cytochrome P450 (CYP). ${ }^{4,5}$ The majority (85-95\%) of ingested clopidogrel never enters the complex CYP-mediated bioactivation cascade due to substantial CES1-mediated hydrolysis of the parent drug to the inactive clopidogrel carboxylic acid, and the remaining 5-15\% of the clopidogrel dose is subject to metabolic activation via a twostep oxidative process mediated by multiple CYP enzymes. ${ }^{4-6}$ In addition, CES1 is also involved in hydrolysis of both the intermediate metabolite 2-oxo-clopidogrel and the 5-thiol clopidogrel active metabolite (clopidogrel-AM). These multiple roles of CES1 suggest that it is a primary metabolic determinant of clopidogrel bioactivation. ${ }^{5-8}$

The majority of studies assessing the importance of genetic variants and drug-drug interactions (DDIs) in the clinical safety and efficacy of clopidogrel have focused on the CYP system, e.g., CYP2C19 gene variants, and the potential influence of cotreatment with statins, proton pump inhibitors, and calcium channel blockers. ${ }^{3,9,10}$ Although significant variability is known to exist in the expression and activity of CES1, including CES1 singlenucleotide polymorphisms associated with markedly impaired hydrolytic activity of CES1, only recently has there been documentation that these CES1 variants are associated with significantly higher exposure to clopidogrel-AM and enhanced platelet inhibition in patients treated with clopidogrel. ${ }^{7,11}$

Angiotensin-converting enzyme inhibitors (ACEIs) are mainstay medications after MI, and most are thought to be substrates for CES1. ${ }^{12,13}$ ACEIs are hence routinely coprescribed with clopidogrel, and we therefore hypothesized that ACEIs, 
when coadministered with clopidogrel, have a high likelihood of competing with clopidogrel for the catalytic site of CES1. This competition could lead to a shunting of a larger fraction of absorbed clopidogrel directly to CYP-mediated activation, ultimately increasing the systemic exposure to clopidogrel-AM, producing greater platelet inhibition and an increased risk of bleeding. To our knowledge, this potential DDI has not been examined previously, and large randomized clinical trials for such purposes would be extremely costly and are unlikely to be performed in the postmarketing period. Other prospective studies, e.g., of ex vivo platelet activation in healthy volunteers receiving these drugs alone or in combination, could provide surrogate end points, but their clinical relevance might be open to question. In this context, a strategy was devised that links the results of targeted in vitro DDI studies to targeted pharmacoepidemiological studies of large populations of real-world patients. Combining these two methodologies, which examine the DDI potential from opposite ends of the "spectrum" of DDI assessment and detection, may represent a useful study paradigm for assessment of the occurrence and clinical relevance of DDIs. Therefore, to potentially provide a proof of concept for this strategy, we investigated the effects of ACEIs on clopidogrel bioactivation in vitro, in tandem with an examination of the nationwide Danish registries to assess the effects of ACEIs on bleeding in patients with MI treated or not treated with clopidogrel.

\section{RESULTS}

\section{In vitro substudy}

The p-nitrophenyl acetate (PNPA) incubation study demonstrated that the four tested ACEIs exhibited varying degrees of inhibitory effects on CES1-mediated PNPA hydrolysis following incubation with $s 9$ fractions prepared from the CES1-transfected human embryonic kidney (HEK)-293 cells (Figure 1a). The calculated $\mathrm{IC}_{50}$ (concentration at which $50 \%$ of enzymatic activity is inhibited) value of enalapril was $447.6 \pm 27.3 \mu \mathrm{mol} / \mathrm{l}$, whereas $50 \%$ inhibition of PNPA hydrolysis was not achieved after incubation with the remaining ACEIs, even at the highest concentration tested $(1,000 \mu \mathrm{mol} / \mathrm{l})$. Further in vitro studies using human liver $\mathrm{s} 9$ fractions were conducted to evaluate the effect of ACEIs on the bioactivation of clopidogrel (Figure 1b,c). Among the tested ACEIs, trandolapril and enalapril were found to significantly increase the formation of 2-oxo-clopidogrel and clopidogrel-AM. In addition, coincubation of clopidogrel with ramipril and perindopril enhanced the formation of clopidogrel-AM, although the increase was not statistically significant under the current experimental conditions. Bis(4-nitrophenyl) phosphate (BNPP), a potent CES1 inhibitor, was included in the study as a positive control. As expected, BNPP significantly increased the production of 2-oxo-clopidogrel and clopidogrel-AM via inhibition of CES1mediated hydrolysis.

\section{Epidemiological substudy}

Study population. We identified a total of 97,528 patients with a first-time MI in the period 2000-2010. Of these, 25,001

\section{Study Highlights}

\section{WHAT IS THE CURRENT KNOWLEDGE ON THE TOPIC?}

Clopidogrel is a prodrug, the majority of which is hydrolyzed to an inactive metabolite by hepatic CES1. This enzyme also activates most ACEls.

\section{WHAT QUESTION DID THIS STUDY ADDRESS?}

$\checkmark$ We examined whether ACEls increased clopidogrel bioactivation in vitro and whether cotreatment with ACEls and clopidogrel was associated with increased risk of clinically significant bleeding in a nationwide pharmacoepidemiological study.

\section{WHAT THIS STUDY ADDS TO OUR KNOWLEDGE}

$\checkmark$ ACEls increased formation of clopidogrel active metabolite in vitro, and cotreatment with these two agents was associated with increased risk of bleeding in patients after MI.

\section{HOW THIS MIGHT CHANGE CLINICAL PHARMACOLOGY}

\section{AND THERAPEUTICS}

The results suggest that increased risk of clinically significant bleeding should be considered in patients cotreated with clopidogrel and ACEls. The combination of targeted in vitro investigations and pharmacoepidemiological studies may represent a useful paradigm for assessment of DDIs.

died or had an event within the 7-day qualifying period and 1,593 were younger than 30 years. Therefore, a total of 70,934 were enrolled in the study. In this population, 29,043 (40.9\%) received clopidogrel within 7 days of discharge, and of these, 9,069 (31.2\%) received ACEIs at baseline. Of the remaining 41,891 (59.1\%) who did not receive clopidogrel, 6,593 (15.7\%) received ACEIs. Baseline characteristics of the study population at time of inclusion are shown in Table 1. In general, subjects who did not receive clopidogrel were in lower income groups, had more comorbidity, and received more concomitant medications, except for $\beta$-blockers, aspirin, and statins. A larger percentage $(63.5 \%)$ of patients treated with PCI received clopidogrel, as compared with the group that did not undergo PCI (36.3\%). Independent of clopidogrel treatment, individuals treated with ACEIs had higher comorbidity and received more concomitant medication at baseline. During the follow-up period, 4,799 clopidogrel-treated subjects and 11,259 patients without clopidogrel treatment changed status from no ACEI treatment to ACEI treatment. The distribution of individual ACEIs used in the study population is shown in Table 2. Of note, ramipril, enalapril, trandolapril, and perindopril comprised $>95 \%$ of the ACEIs used, with enalapril and trandolapril accounting for almost $50 \%$ of all ACEIs used during the study period.

Bleeding, cardiovascular death, MI, stroke, and all-cause mortality during 1 year of follow-up. Crude incidence rates of study end points per 100 patient-years in subjects who received clopidogrel or ACEIs as single therapies or in combination, after first-time MI during 1 year of follow-up are shown in Supplementary Table S1 online. As shown in Table 3, in the 

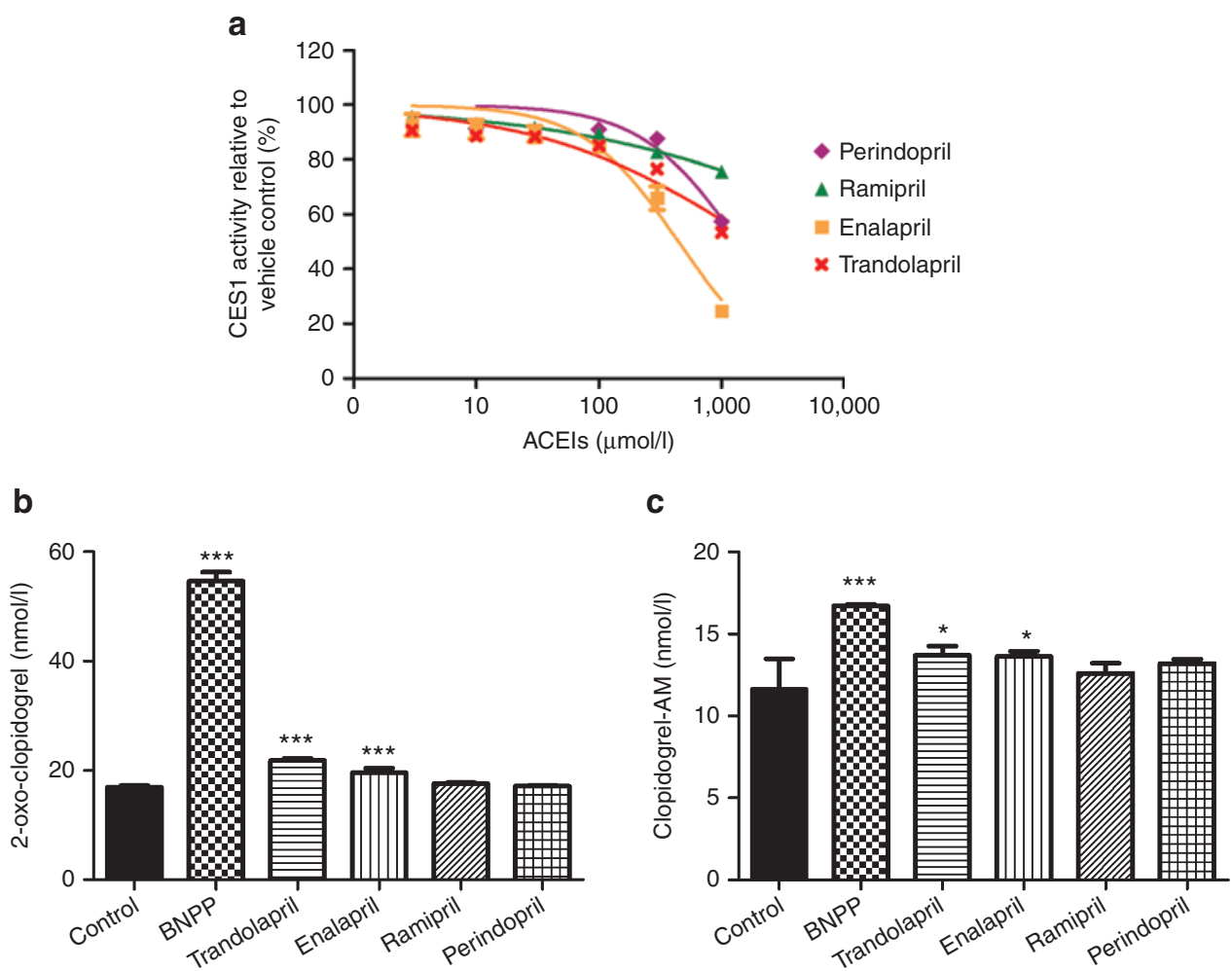

Figure 1 Clopidogrel bioactivation in vitro. (a) Effects of the angiotensin-converting enzyme inhibitors (ACEls) trandolapril, enalapril, ramipril, and perindopril on carboxylesterase 1 (CES1) activity, determined by $p$-nitrophenyl acetate (PNPA) hydrolysis in CES1-transfected HEK-293 cells and formation of (b) 2-oxoclopidogrel and (c) clopidogrel-AM (active metabolite) after incubation of clopidogrel with human liver s9 fractions. Data are presented as means \pm SD $(n=3)$. BNPP, bis(4-nitrophenyl) phosphate; HEK, human embryonic kidney. ${ }^{*} P<0.05$ and ${ }^{* * *} P<0.001$ vs. control.

adjusted Cox proportional hazard analyses, treatment with ACEIs in clopidogrel-treated patients was associated with a nominally increased risk of bleeding (hazard ratio (HR): 1.10; 95\% confidence interval $(\mathrm{CI}): 0.97-1.25 ; P=0.124)$, whereas in the absence of clopidogrel treatment, ACEIs were associated with a significantly decreased risk of bleeding (HR: 0.90; 95\% CI: 0.81-0.99; $P=0.025)$. The difference between these two HRs, expressed as the hazard rate ratio (HRR), was statistically significant (HRR: 1.27; 95\% CI: $1.09-1.49 ; P=0.002$ ), indicating an effect modification of concomitant treatment with ACEIs and clopidogrel on bleeding. This association was reproducible in all examined subgroups (Figure 2). As shown in Table 3 and Figure 3, similar results were found for the secondary end points of cardiovascular death (HR: 1.29; 95\% CI: 1.14-1.44; $P<0.001$ ), all-cause mortality (HR: 1.14; 95\% CI: 1.04-1.25; $P<0.001$ ), and the composite of cardiovascular death, MI, or stroke (HR: 1.12; 95\% CI: 1.06-1.19; $P<0.001$ ), although no differences in risk were found for MI (HRR: 0.95; 95\% CI: $0.87-1.03 ; P=0.223$ ) or stroke (HRR: 1.10 ; 95\% CI: $0.89-1.36 ; P=0.376)$. When analyses of bleeding were done on the basis of individual ACEIs, the interaction with clopidogrel tended to be absent in the group of patients receiving captopril and lisinopril, the only two ACEIs that are not metabolized by CES1 (Figure 4). In addition, we found no interaction between ACEI treatment and any of the explanatory covariates for the primary end point of bleeding.

\section{DISCUSSION}

The current proof-of-concept study, with a combination of in vitro and pharmacoepidemiological investigations, provided two important new insights. First, the in vitro assessments showed that all tested ACEIs increased the bioactivation of clopidogrel in human liver $\mathrm{s} 9$ fractions and that significantly increased concentrations of clopidogrel-AM were achieved following coincubation of clopidogrel with trandolapril and enalapril. Second, the clinical importance of these in vitro findings was apparently borne out in a nationwide pharmacoepidemiological study of patients discharged after first-time MI and followed for 1 year, in which treatment with ACEIs was associated with significantly increased risk of clinically important bleeding in patients receiving clopidogrel. Cotreatment with ACEIs and clopidogrel was also linked to increased risk of cardiovascular death and all-cause mortality, and this association with increased risk of bleeding was consistent in all examined subgroups. To our knowledge, these data, obtained with the use of an approach that combined in vitro and pharmacoepidemiological investigations, are the first to suggest a clinically significant ACEI-clopidogrel interaction. The in vitro demonstration of the CES1-centered mechanism of interaction provides a compelling rationale for the pharmacoepidemiological results, and this combined approach may represent a useful paradigm for evaluation of suspected DDIs in the postmarketing period, which might be applied to various drug combinations in use. 
Table 1 Baseline characteristics of patients with first-time myocardial infarction who survived 7 days from discharge

\begin{tabular}{|c|c|c|c|c|}
\hline & \multicolumn{2}{|c|}{ Clopidogrel $(n=29,043)$} & \multicolumn{2}{|c|}{ No clopidogrel $(n=41,891)$} \\
\hline & No ACEI $(n=19,974)$ & ACEI $(n=9,069)$ & No ACEI $(n=35,298)$ & ACEI $(n=6,593)$ \\
\hline & $n=15,175^{\mathrm{a}}$ & $n=13,868^{\mathrm{a}}$ & $n=24,039^{a}$ & $n=17,852^{\mathrm{a}}$ \\
\hline Men (\%) & $13,375(67.0)$ & $6,262(69.1)$ & $20,417(57.8)$ & $3,905(69.2)$ \\
\hline Age (mean (SD) years) & $63.9( \pm 12.5)$ & $63.6( \pm 12.4)$ & $67.7( \pm 13.4)$ & $69.2( \pm 12.3)$ \\
\hline Age (mean (SD) years) & $70.4( \pm 13.4)$ & $70.4( \pm 13.1)$ & $75.0( \pm 13.0)$ & $75.8( \pm 11.6)$ \\
\hline \multicolumn{5}{|l|}{ Year of inclusion } \\
\hline 2000-2004 (\%) & $6,282(31.4)$ & $2,929(32.2)$ & $20,406(56.8)$ & $4,795(72.8)$ \\
\hline 2005-2010 (\%) & $13,629(68.6)$ & $6,140(67.8)$ & $14,892(43.2)$ & $1,798(27.2)$ \\
\hline \multicolumn{5}{|l|}{ Income group } \\
\hline $\mathrm{PCl}(\%)$ & $5,607(28.1)$ & $2,048(22.6)$ & $4,086(11.6)$ & $316(4.8)$ \\
\hline \multicolumn{5}{|l|}{ Comorbidity } \\
\hline Shock (\%) & $122(0.6)$ & $58(0.6)$ & $703(2.0)$ & $62(0.9)$ \\
\hline DM with complications (\%) & $667(3.3)$ & $279(3.1)$ & $1,979(5.6)$ & $374(5.7)$ \\
\hline Peptic ulcer (\%) & $178(0.9)$ & $63(0.7)$ & $784(2.2)$ & $99(1.5)$ \\
\hline Pulmonary edema (\%) & $96(0.5)$ & $57(0.6)$ & $441(1.3)$ & $123(1.9)$ \\
\hline Peripheral vascular disease (\%) & $381(1.9)$ & $127(1.4)$ & $1,143(3.2)$ & $160(2.4)$ \\
\hline Cerebrovascular disease (\%) & $597(3.0)$ & $241(2.7)$ & $2,266(6.4)$ & $318(4.8)$ \\
\hline Thiazide (\%) & $622(3.3)$ & $455(5.0)$ & $824(2.3)$ & $384(5.8)$ \\
\hline Spironolactone (\%) & $373(1.9)$ & $594(6.6)$ & $807(2.3)$ & $965(14.6)$ \\
\hline Aspirin (\%) & $14,284(71.5)$ & $7,383(81.4)$ & $10,063(28.5)$ & $4,319(65.5)$ \\
\hline Vitamin K antagonist (\%) & $278(1.4)$ & $250(2.8)$ & $694(2.0)$ & $563(8.5)$ \\
\hline Statin (\%) & $14,299(71.6)$ & $7,440(82.0)$ & $6,300(17.9)$ & $2,959(44.9)$ \\
\hline$\beta$-Blocker (\%) & $14,584(73.0)$ & $7,495(82.6)$ & 10,795 (30.6) & $4,660(70.7)$ \\
\hline CCB (\%) & $1,517(7.6)$ & $510(5.6)$ & $1,787(5.1)$ & $518(7.9)$ \\
\hline Digoxin (\%) & $389(2.0)$ & $272(3.0)$ & $1,050(3.0)$ & $600(9.1)$ \\
\hline Glucose-lowering drug (\%) & $566(2.8)$ & 441 (4.9) & $747(2.1)$ & $423(6.4)$ \\
\hline
\end{tabular}

$\mathrm{ACEl}$, angiotensin-converting enzyme inhibitor; $\mathrm{CCB}$, calcium channel blocker; $\mathrm{DM}$, diabetes mellitus; $\mathrm{PCl}$, percutaneous coronary intervention.

aNumber of patients 1 year after inclusion.

Clopidogrel is a prodrug undergoing substantial biotransformation catalyzed by hepatic CES1, which is responsible for $80-95 \%$ of the total hydrolytic activity in the human liver. ${ }^{7}$ CES1 catalyzes the hydrolysis of ester- and amide-containing endogenous substrates, toxins, and various drugs to their respective free acids, and evolving evidence suggests that the importance of CES1 in human drug metabolism may have been somewhat overlooked relative to CYP enzymes. ${ }^{5,14,15}$ After administration of the prodrug, the majority of absorbed clopidogrel is rapidly hydrolyzed by CES1 to the inactive carboxylic acid metabolite, and only the remaining $5-15 \%$ is subjected to CYP-mediated bioactivation. ${ }^{6,14}$ Furthermore, CES1 hydrolyzes a proportion of 
Table 2 Distribution of ACEls at baseline and after 1 year

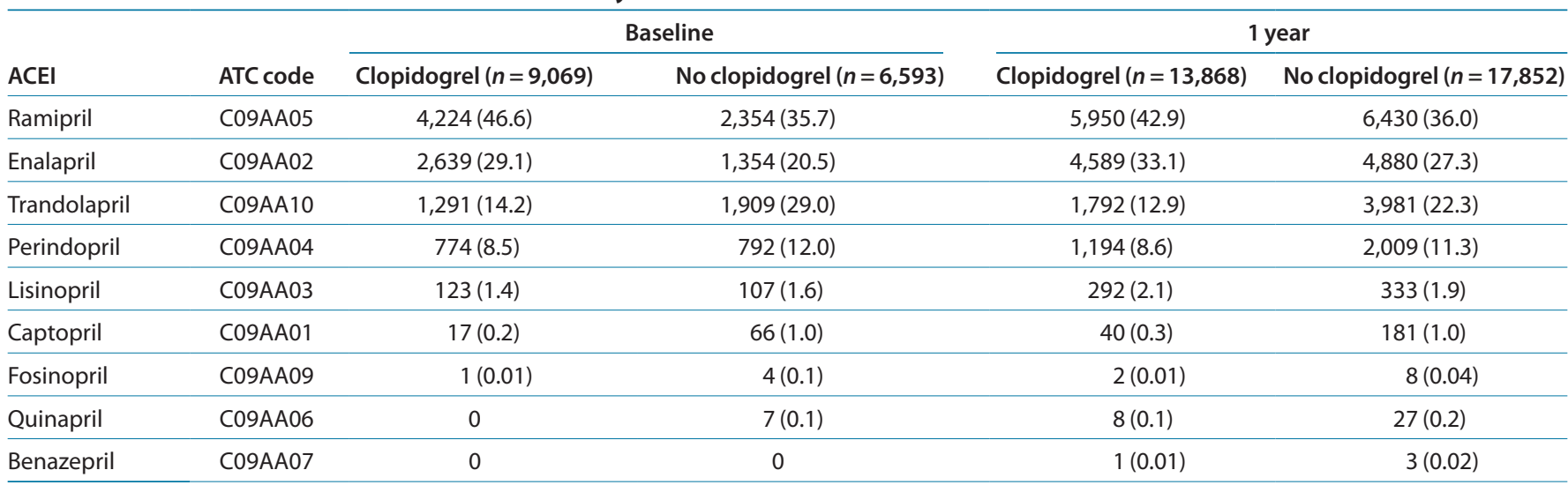

Data are given as $n(\%)$.

ACEl, angiotensin-converting enzyme inhibitor; ATC, Anatomical Therapeutic Chemical Classification System.

Table 3 Risk associated with ACEls and clopidogrel as single therapies and in combination

\begin{tabular}{|c|c|c|c|c|c|c|c|c|}
\hline & \multicolumn{3}{|c|}{ Clopidogrel $(n=29043)$} & \multicolumn{3}{|c|}{ No clopidogrel $(n=41891)$} & \multicolumn{2}{|c|}{$\begin{array}{l}\text { Likelihood for difference with } \\
\text { ACEl treatment }\end{array}$} \\
\hline & No ACEI & ACEI & & No ACEI & ACEI & & & \\
\hline Bleeding & 1.00 & $1.10(0.97-1.25)$ & 0.124 & 1.00 & $0.90(0.81-0.99)$ & 0.025 & $1.27(1.09-1.49)$ & 0.002 \\
\hline $\mathrm{Ml}$, stroke, or CV death & 1.00 & $1.12(1.06-1.19)$ & $<0.001$ & 1.00 & $1.03(0.99-1.07)$ & 0.162 & $1.09(1.02-1.17)$ & 0.014 \\
\hline CV death & 1.00 & $1.29(1.14-1.44)$ & $<0.001$ & 1.00 & $0.85(0.80-0.90)$ & $<0.001$ & $1.49(1.32-1.70)$ & $<0.001$ \\
\hline All-cause mortality & 1.00 & $1.14(1.04-1.25)$ & 0.007 & 1.00 & $0.81(0.77-0.85)$ & $<0.001$ & $1.42(1.28-1.57)$ & $<0.001$ \\
\hline
\end{tabular}

Results from Cox proportional hazard models adjusted for age, gender, percutaneous coronary intervention, income, concomitant medication, and comorbidity.

$\mathrm{ACEl}$, angiotensin-converting enzyme inhibitor; $\mathrm{Cl}, 95 \%$ confidence interval; $\mathrm{CV}$, cardiovascular; $\mathrm{HR}$, hazard ratio; $\mathrm{HRR}$, hazard rate ratio; $\mathrm{MI}$, myocardial infarction.

*P value for interaction between ACEls and clopidogrel.

a 2-oxo-clopidogrel intermediate in the complex CYP oxidation scheme, as well as hydrolyzing the unstable 5-thiol clopidogrelAM itself. ${ }^{7,8}$ In addition to its role in inactivation of clopidogrel, CES1 is involved in metabolism of other drugs, and previous interest has focused on methylphenidate, oseltamivir, and ACEIs. ${ }^{14,15}$ Specifically, most ACEIs are esterified prodrugs that are likewise activated by hydrolysis, and CES1 has conclusively been shown to govern hydrolysis of trandolapril; accumulating evidence has suggested that CES1 is responsible for activation of most other ACEIs, apart from captopril and lisinopril, which are devoid of ester bonds. ${ }^{12,13,15-19}$

Platelet inhibition in response to clopidogrel is highly variable, and clopidogrel resistance is associated with ischemic events, including stent thrombosis after PCI, whereas increased exposure to clopidogrel can result in bleeding. ${ }^{20,21}$ Hithertoidentified genetic variants (e.g., CYP2C19*2) and demographic and/or clinical factors influencing clopidogrel disposition and pharmacodynamics explain only a small part of the variability in the response to clopidogrel. ${ }^{9,22,23}$ Recently, however, single-nucleotide polymorphisms in CES1 were found to have marked effects on clopidogrel bioactivation. Specifically, in vitro studies have documented that the catalytic activity of a CES1 p.Gly143Glu variant was essentially abolished in terms of catalyzing the hydrolysis of clopidogrel and 2-oxo-clopidogrel. ${ }^{12}$ Furthermore, subjects with this variant were found to have high on-treatment levels of the clopidogrel-AM and increased platelet inhibition as assessed by platelet function assays. ${ }^{11}$ This CES1 gene variant has previously been associated with loss of CES1's ability to hydrolyze ACEIs (i.e., trandolapril). ${ }^{12}$ Because alterations of CES1 activity clearly influence clopidogrel bioactivation and platelet inhibitory effects, and because our in vitro results in the HEK-293 cell model system support the hypothesis that ACEIs inhibits CES1 catalytic activity (Figure 1a), we hypothesized that coincubation of ACEIs and clopidogrel could increase formation of clopidogrel-AM in human liver s9 fractions. Indeed, this was found to be the case (Figure 1b,c), setting the stage for our pharmacoepidemiological substudy, in which cotreatment with the two agents was associated with increased risk of clinically important bleeding.

In the current study, the largest increase in risk of bleeding associated with the ACEI-clopidogrel combination was observed in patients included before 2004 and in those who 


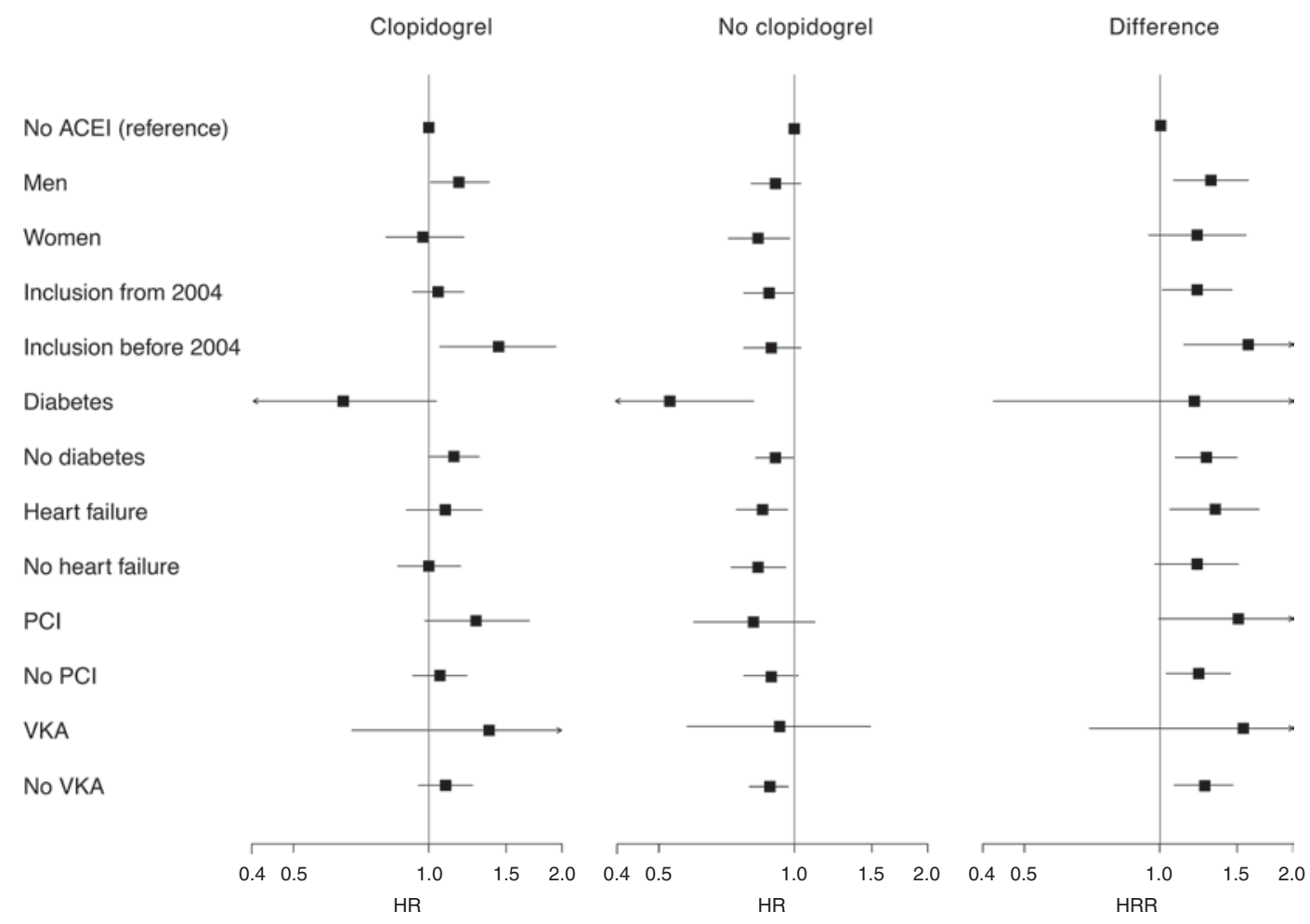

Figure 2 Risk of bleeding in selected subpopulations. Results from adjusted multivariate Cox proportional hazard analyses. Error bars illustrate $95 \%$ confidence intervals. Hazard rate ratios (HRRs) illustrate difference in risk for treatment with angiotensin-converting enzyme inhibitors (ACEls) between the groups treated with or without clopidogrel. HR, hazard ratio; $\mathrm{PCl}$, percutaneous coronary intervention; VKA, vitamin K antagonist.
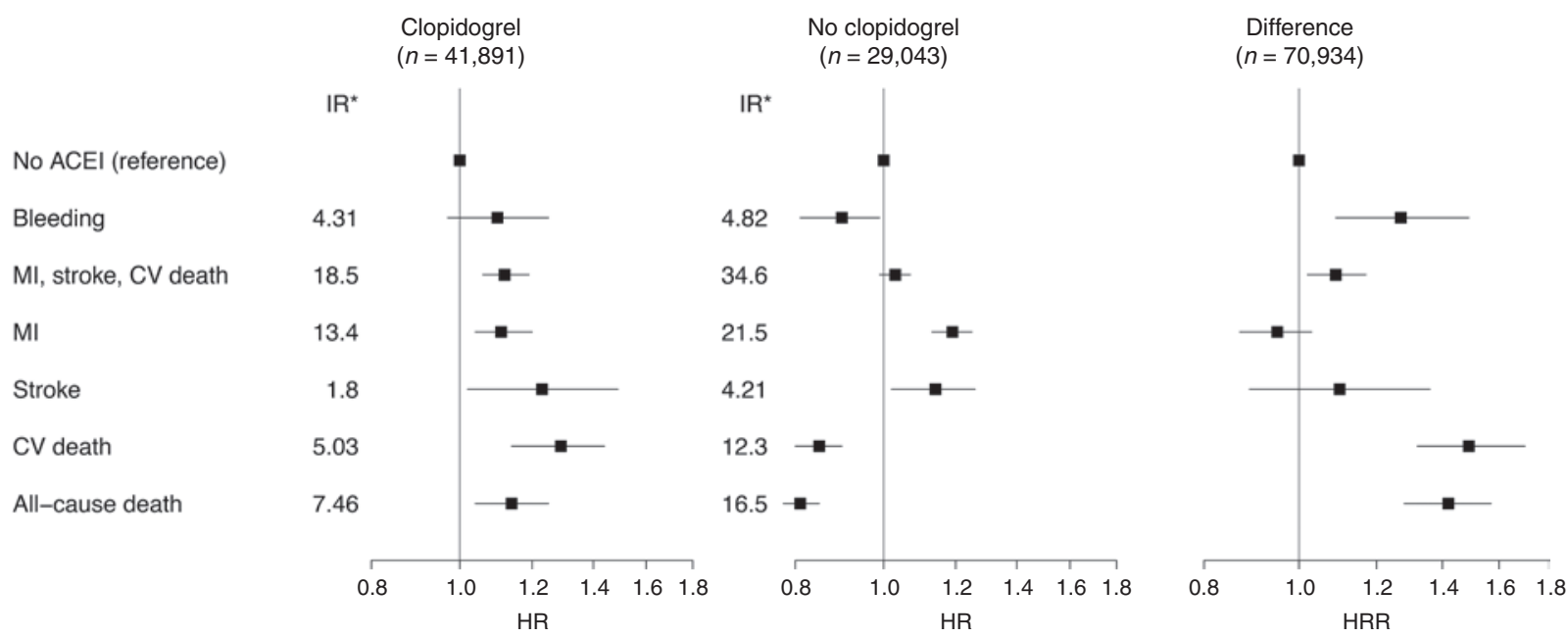

Figure 3 Risk of bleeding and secondary study outcomes. Results from adjusted multivariate Cox proportional hazard analyses. Error bars illustrate $95 \%$ confidence intervals. Hazard rate ratios (HRRs) illustrate difference in risk for treatment with angiotensin-converting enzyme inhibitors (ACEls) between the groups with or without clopidogrel. CV, cardiovascular; HR, hazard ratio; IR, incidence rate; MI, myocardial infarction. ${ }^{*}$ Crude IRs per 100 patient-years.

underwent PCI (Figure 2). Before 2004, treatment with clopidogrel after MI was not firmly established in the Danish clinical guidelines, and during this period, there was probably less difference in confounding variables between patients with vs. those without clopidogrel; i.e., the more pronounced bleeding signal here is arguably supportive of our hypothesis. Bleeding represents the most common complication after PCI, and in accord with our current findings, these patients were expected to bleed more with increased exposure to clopidogrel. On the other hand, absence of increased bleeding in patients with diabetes who are treated concurrently with ACEIs and clopidogrel is in agreement with the increased prevalence of high platelet reactivity in these subjects; i.e., increased exposure to clopidogrel may not be sufficient to cause significant bleeding. ${ }^{24,25}$ In addition to increased risk of bleeding, the ACEI-clopidogrel combination was also associated with higher risk of cardiovascular death, allcause mortality, and a composite of cardiovascular death, MI, or stroke, and the last was primarily driven by cardiovascular 

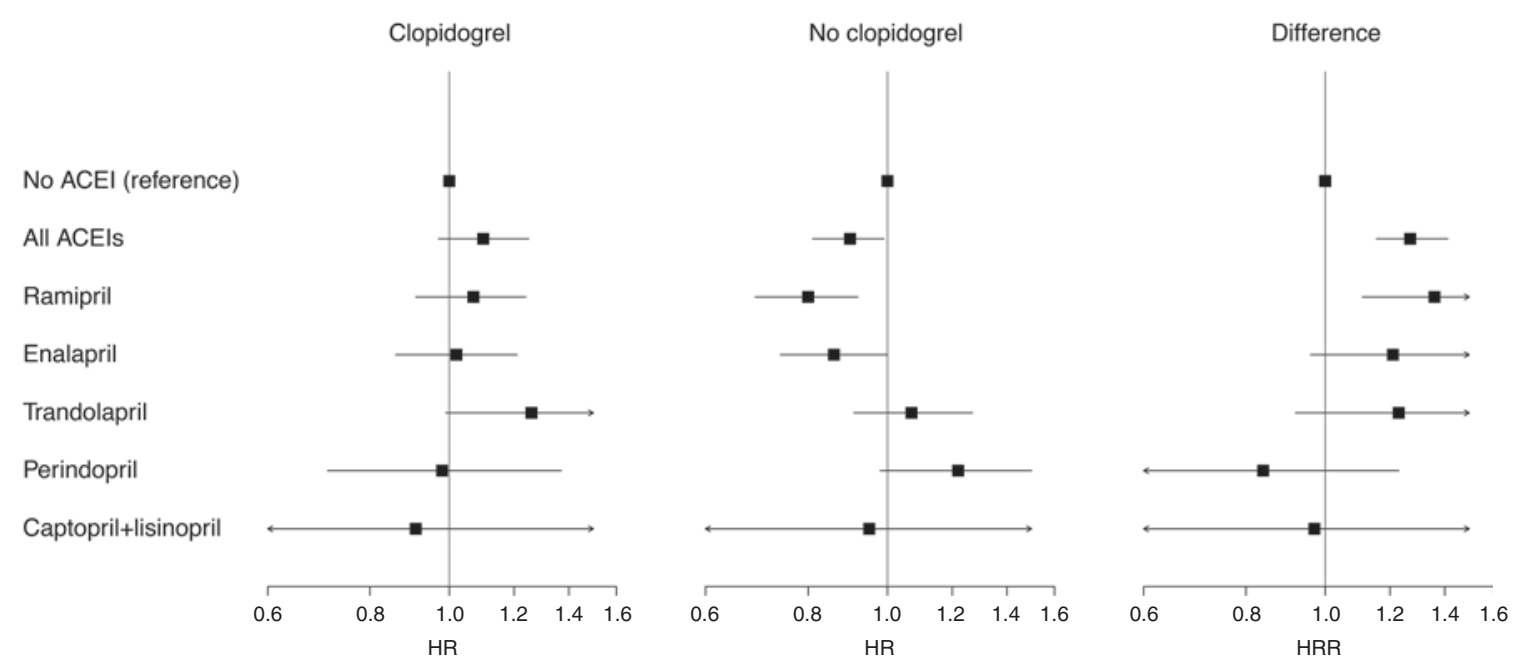

Figure 4 Risk of bleeding according to individual angiotensin-converting enzyme inhibitors (ACEls) and clopidogrel treatment. Results from adjusted timedependent Cox proportional hazard analyses. Error bars illustrate $95 \%$ confidence intervals. Hazard rate ratios (HRRs) illustrate difference in bleeding risk for treatment with ACEls between the groups with and without clopidogrel. HR, hazard ratio. $P$ values for the difference between patients treated or not treated with clopidogrel were $0.002,0.004,0.101,0.159,0.373$, and 0.943 for all ACEls, ramipril, enalapril, trandolapril, perindopril, and captopril+lisinopril, respectively.

deaths (Table 3; Figure 2). Although increased risk of ischemic events in the face of more bleeding with the drug combination would seem counterintuitive, it is well established that bleeding in post-MI patients is associated with death, stroke, and MI due to complications triggered by the bleeding itself and probable discontinuation of antithrombotic agents in the case of clinically significant bleeding, as well as secondary increased risk of ischemic events.

As indicated above, the significant interindividual variability of CES1 activity is defined, in part, by variation of the CES1 gene. This gene is located on chromosome 16 , and different structural haplotypes and sequence variants have been reported, with frequencies being dependent on the ethnic background. ${ }^{26,27}$ The minor allele frequency of p.Gly143Glu was first determined to be 3.7, 4.3, 2.0, and $0 \%$ in white, black, Hispanic, and Asian populations, respectively, whereas frequencies of 0.6 and $0.85 \%$ were recently reported in an Amish population and US patients with coronary artery disease, respectively. ${ }^{11,28}$ Subjects with p.Gly143Glu or other CES1 variants are probably prone to bleeding when treated with clopidogrel, irrespective of ACEI cotreatment, ${ }^{6,11}$ but because of their relatively low prevalence, these variants are unlikely to have considerably skewed our results.

In addition to clopidogrel and ACEIs, other drugs with cardiovascular indications (e.g., dabigatran, simvastatin, and carvedilol) and various other medications (including methylphenidate, oseltamivir, flumazenil, mycophenolate mofetil, oxybutynin, and rufinamide) are also hydrolyzed by CES1, but as yet, potential DDIs involving combinations of these agents have not been assessed or reported. ${ }^{14,15,29-32}$ Importantly, aspirin and newer platelet adenosine diphosphate receptor antagonists, including prasugrel and ticagrelor, are not metabolized by CES1 (aspirin and prasugrel are hydrolyzed by CES2, a distinct CES predominantly present in the intestine, and ticagrelor is not a prodrug) and are therefore not subject to the DDI mechanism described above. ${ }^{5,6,33}$ The current results therefore raise the intriguing possibility that the relative increase in bleeding risk observed with prasugrel and ticagrelor in randomized trials that established improved efficacy of these agents as compared with clopidogrel in patients with acute coronary syndromes may have been even higher in absence of the large representation of individuals treated with ACEIs in these studies. ${ }^{34,35}$

\section{Limitations}

In vitro studies cannot accurately simulate in vivo conditions. A relatively high concentration of clopidogrel $(20 \mu \mathrm{mol} / \mathrm{l})$, which is substantially greater than what can be achieved in vivo, was used in the current study in order to produce measurable clopidogrel-AM in the in vitro system. However, such high substrate concentration may have attenuated the inhibitory effect of ACEIs on CES1 in the current study. Therefore, it is possible that the CES1-mediated DDI between ACEIs and clopidogrel can be more significant in vivo than that observed in the in vitro study. Epidemiological observational studies are inherently at risk of unmeasured confounders and do not allow for causal conclusions. Indeed, there were baseline differences between our patient groups. Importantly, by using the combined data from patients with vs. without clopidogrel and treated (or not) with ACEIs, our statistical model also provided adjustment for confounders related to treatment with ACEIs and thereby allowed for estimation of the HRR for interaction between ACEIs and clopidogrel, i.e., the effect modification on bleeding that was most likely to be the result of the DDI. Notwithstanding statistical adjustments, however, unmeasured confounders may have affected these groups differently, and controls treated with prasugrel or ticagrelor may have provided further insights. Only bleedings that require hospitalization or fatal bleedings are registered in the Danish registries, and we cannot account for minor bleeding events. Baseline medication was evaluated by retrieved prescriptions within 7 days after discharge. Therefore, we did not consider alterations in medication during the year of follow-up, 
except for use of ACEIs, which was assessed in a time-dependent manner. In our main analyses, all ACEIs were considered together, although captopril and lisinopril are not metabolized by CES1. ${ }^{12,15-19}$ However, these two ACEIs comprised $<5 \%$ of ACEIs used in the study and are therefore unlikely to have significantly diluted the results (Table 2). Although the analyses applied to the individual ACEIs had reduced statistical power, the increased risk of bleeding associated with other examined ACEIs tended to be absent with captopril and lisinopril, which is in agreement with our study hypothesis (Figure 4). In addition, the influences of other drugs that have been proposed to modify clopidogrel efficacy and safety as a result of CYP-mediated interactions, e.g., selected statins, proton pump inhibitors, and calcium channel blockers, were not included as part of the current analyses. ${ }^{9,13,15,29}$

\section{Conclusions}

To our knowledge, this is the first report to document an ACEIclopidogrel interaction. We found that, in vitro, coincubation with ACEIs increased clopidogrel bioactivation, and concomitant treatment with ACEIs in patients who received clopidogrel after first-time MI was associated with increased risk of clinically important bleeding. The results raise concerns about concomitant treatment with clopidogrel and ACEIs. The dual in vitro and pharmacoepidemiological approach may prove to be a useful study paradigm for assessment of suspected DDIs.

\section{METHODS}

\section{In vitro substudy}

Materials. Enalapril maleate, perindopril erbumine, moexipril hydrochloride, PNPA, the derivatizing agent 2-bromo-3'-methoxyacetophenone (MPB), and the CES1 inhibitor BNPP were purchased from SigmaAldrich (St Louis, MO). p-Nitrophenol was obtained from TCI America (Portland, OR). Fosinopril sodium salt, ramipril, $S-(+)$-clopidogrel, 2-oxo-clopidogrel, MPB-derivatized clopidogrel active metabolites (cisclopidogrel thiol metabolite and clopidogrel-AM), and the internal standard d4-clopidogrel carboxylate were from Toronto Research Chemicals (Toronto, ON, Canada). Pooled human liver s9 fraction (HLS9) and the RapidStart nicotinamide adenine dinucleotide phosphate (NADPH) Regenerating System were purchased from XenoTech (Lenexa, KS). All other chemicals and reagents were of the highest analytical grade commercially available.

Inhibitory effect of ACEIs on CES1-mediated PNPA hydrolysis. The model CES1 substrate PNPA and the HEK-293 cells stably expressing wild-type CES1 - and no other major drug-metabolizing enzymes, including CYP enzymes - were used to assess the potential inhibitory effects of trandolapril, enalapril, ramipril, and perindopril on CES1 activity according to methods described previously. ${ }^{12,28}$ The final concentrations of the wildtype CES1 cell s9 proteins and PNPA were $25 \mu \mathrm{g} / \mathrm{ml}$ and $100 \mu \mathrm{mol} / \mathrm{l}$, respectively. The final concentrations of each tested ACEI ranged from 0 to $1,000 \mu \mathrm{mol} / \mathrm{l}$. The reaction mixtures contained various concentrations of dimethyl sulfoxide or methanol to improve solubility of ACEIs. Potential effects of the organic solvents on CES1 activity were controlled for by including vehicle controls. All experiments were carried out in triplicate.

Influence of ACEIs on clopidogrel activation in human liver 99 fractions. Pooled human liver $\mathrm{s} 9$ fractions were preincubated with the RapidStart NADPH Regenerating System (1 mmol/l yeast glucose-6-phosphate dehydrogenase, $1 \mathrm{mmol} / \mathrm{l}$ nicotinamide adenine dinucleotide phosphate, and $5 \mathrm{mmol} / \mathrm{l}$ glucose-6-phosphate) in the absence or presence of trandolapril, enalapril, ramipril, perindopril, and the known CES1 inhibitor BNPP at $37^{\circ} \mathrm{C}$ for $10 \mathrm{~min}$. The reaction was initiated by adding the substrate clopidogrel. The final concentrations of the liver $\mathrm{s} 9$ fractions and clopidogrel in the reaction system were $2 \mathrm{mg} / \mathrm{ml}$ and $20 \mu \mathrm{mol} / \mathrm{l}$, respectively. The concentration of all tested ACEIs was $50 \mu \mathrm{mol} / \mathrm{l}$, and the concentration of BNPP was $10 \mu \mathrm{mol} / \mathrm{l}$. The final volume of the reaction system was $100 \mu \mathrm{l}$. The incubation time was $2 \mathrm{~h}$, which was based on our previous studies that indicated that the maximum concentrations of formed clopidogrel-AM were achieved around $2 \mathrm{~h}$ following the initiation of incubation. ${ }^{7}$ The reaction was terminated by adding a twofold volume of acetonitrile containing the internal standard d4-clopidogrel carboxylate $(50 \mathrm{ng} / \mathrm{ml})$ and the derivatizing reagent MPB $(5 \mathrm{mmol} / \mathrm{l})$. MPB was used to form the stable MPB derivative of unstable clopidogrel-AM to facilitate analysis. The mixtures were left undisturbed at room temperature for $10 \mathrm{~min}$ to allow the derivatization reaction to be completed. ${ }^{36,37}$ Samples were then centrifuged at $17,000 \times g$ at $4^{\circ} \mathrm{C}$ for 20 min to remove precipitated proteins. The resulting supernatant was collected for liquid chromatography-tandem mass spectrometry analysis. The concentrations of 2-oxo-clopidogrel and clopidogrel-AM were determined by a validated liquid chromatography-tandem mass spectrometry assay described previously. ${ }^{7}$

\section{Pharmacoepidemiological substudy}

The Danish Registries. As a data source, we used the Danish nationwide administrative registries. In Denmark, each citizen has a unique and permanent civil registration number that enables individual-level linkage of information from nationwide administrative registries. The National Patient Registry holds information on all admissions to Danish hospitals since 1978 according to the International Classification of Diseases (ICD) codes. $^{38}$ Information on all prescriptions dispensed from Danish pharmacies since 1995 is enumerated in the Danish Registry of Medicinal Product Statistics (prescription registry) according to the international Anatomical Therapeutic Chemical Classification System. ${ }^{39}$ Primary and contributing causes of death are registered in the National Causes of Death Registry. Information on birth date, vital statistics, gender, annual income, and migration were obtained from the Central Person Registry.

Study population and outcomes. All patients $\geq 30$ years of age hospitalized with a first-time MI in the period 2000-2010 were identified from the National Patient Registry. Treatments with clopidogrel (B01AC04) and ACEIs (C09A) were determined by prescription claims within 7 days after discharge. Previous studies have documented that adherence to clopidogrel is high in this population, with 1-year prescription continuation rates of $89 \%$ after $2004 .^{40}$ ACEIs were divided into the individual agents marketed in Denmark during the study period, i.e., captopril (C09AA01), enalapril (C09AA02), lisinopril (C09AA03), perindopril (C09AA04), ramipril (C09AA05), quinapril (C09AA06), benazepril (C09AA07), fosinopril (C09AA09), and trandolapril (C09AA10). Data on individual drug doses were not available in the current data set. To avoid immortal time bias, only patients who survived without experiencing a study outcome the first 7 days after discharge were included in the study. Patients who permanently left the country within the study period were censored at time of emigration. The population was followed for 1 year after discharge.

The primary outcome of the study was clinically significant bleeding, including bleeding requiring hospitalization and fatal bleeding identified through the National Causes of Death Register, i.e., cerebral bleeding (ICD codes I60-62, S06.4-06.6), bleeding from the respiratory tract (J94.2, R04), gastrointestinal bleeding (K25.0, K25.2, K25.4, K26.0, K26.2, K26.4, K27.0, K27.2, K28.0, K28.2, K92.0-92.2), and bleeding from the urinary tract (R31), as defined by either first admission with a diagnosis of nonfatal bleeding or as death from bleeding. ${ }^{41}$ Hospitalization due to bleeding has recently been validated for use in registry studies with positive predictive diagnostic values between 89 and $99 \% .{ }^{42}$ Hospitalizations due to nonfatal bleedings-but that ended with death within a week after admission-were considered fatal bleedings. Secondary outcomes were a composite of cardiovascular death (I00-I99), hospitalization for recurrent MI (I21-I22), or stroke (I63-I66) - the individual components of the combined end point - and all-cause mortality. The MI and stroke diag- 
noses have previously been validated in the National Patient Registry. ${ }^{43,44}$ For patients included in the study who experienced more than one of the above-mentioned adverse events, only the first event that occurred was considered in the data analysis.

Comorbidity, concomitant medication, and socioeconomic status. A comorbidity index was defined by the use of diagnoses at discharge after the index MI, as described in the Ontario acute MI mortality prediction rules. ${ }^{45}$ We further enhanced the power of this index by adding discharge diagnoses from the year before the index MI, as previously described. ${ }^{45,46}$ We also obtained information on claimed prescriptions up to 7 days after discharge for other medications (Table 1). Moreover, we divided patients into five socioeconomic classes using the individual average annual gross income during the 5-year period before study inclusion.

Statistical analyses. All in vitro data are presented as means \pm SDs. In the PNPA hydrolysis study, $\mathrm{IC}_{50}$ values were estimated by fitting the inhibition data to sigmoidal dose-response equations using GraphPad Prism 6.0 software (GraphPad Software, San Diego, CA). The differences between the ACEI-treated and control groups were analyzed using oneway analysis of variance. In the epidemiological study, comparisons of baseline characteristics, including continuous and categorical variables, were made using $\chi^{2}$ test and Students $t$-test, respectively. Treatment with ACEIs was included in the analyses as a time-dependent variable. HRs for outcome variables, as well as likelihood for difference of outcome with ACEI treatment, expressed as HRRs, were determined from Cox proportional hazard models. These models were adjusted for age, gender, calendar year, concomitant medication, comorbidity, income, and PCI. Furthermore, we performed analyses on selected subgroups. In all analyses, $P<0.05$ was considered statistically significant.

Ethics. Ethical approval is not required for retrospective registry studies in Denmark. The study was approved by the Danish Data Protection Agency (2007-58-0015/GEH-2014-012 I-Suite 02720). The work was carried out in accordance with the Uniform Requirements for Manuscripts Submitted to Biomedical Journals (http://www.icmje.org).

SUPPLEMENTARY MATERIAL is linked to the online version of the paper at http://www.nature.com/cpt

\section{ACKNOWLEDGMENTS}

The current study is part of the Individualized Drug Therapy Based on Pharmacogenomics: Focus on Carboxylesterase 1 (INDICES) project, which was funded by grant 10-092792/DSF from the Danish Council for Strategic Research, Programme Commission on Individuals, Disease and Society. G.H.G. is funded by an independent research scholarship from the Novo Nordisk Foundation.

\section{AUTHOR CONTRIBUTIONS}

P.R.H., K.E.K., H.-J.Z., and J.S.M. wrote the manuscript. P.R.H., K.E.K., H-J.Z., G.H.G., and J.S.M. designed the research. P.R.H., K.E.K., H-J.Z., X.W., and J.S.M. performed the research. P.R.H., K.E.K., H-J.Z., X.W., G.H.G., C.T.-P., H.B.R., and J.S.M. analyzed the data.

\section{CONFLICT OF INTEREST}

The authors declared no conflict of interest.

(c) 2014 American Society for Clinical Pharmacology and Therapeutics

1. Anderson, J.L. et al. ACC/AHA 2007 guidelines for the management of patients with unstable angina/non-ST-Elevation myocardial infarction: a report of the American College of Cardiology/American Heart Association Task Force on Practice Guidelines (Writing Committee to Revise the 2002 Guidelines for the Management of Patients With Unstable Angina/Non-ST-Elevation Myocardial Infarction) developed in collaboration with the American College of Emergency Physicians, the Society for Cardiovascular Angiography and Interventions, and the Society of Thoracic Surgeons endorsed by the American Association of Cardiovascular and Pulmonary Rehabilitation and the Society for Academic Emergency Medicine. J. Am. Coll. Cardiol. 50, e1-e157 (2007).

2. O'Gara, P.T. et al. ACCF/AHA 2013 guideline for the management of ST-elevation myocardial infarction: a report of the American College of Cardiology Foundation/American Heart Association Task Force on Practice Guidelines.J. Am. Coll. Cardiol. 61, e78-140.

3. Ahmad, T., Voora, D. \& Becker, R.C. The pharmacogenetics of antiplatelet agents: towards personalized therapy? Nat. Rev. Cardiol. 8, 560-571 (2011).

4. Kazui, M. et al. Identification of the human cytochrome P450 enzymes involved in the two oxidative steps in the bioactivation of clopidogrel to its pharmacologically active metabolite. Drug Metab. Dispos. 38, 92-99 (2010).

5. Hagihara, K. et al. A possible mechanism for the differences in efficiency and variability of active metabolite formation from thienopyridine antiplatelet agents, prasugrel and clopidogrel. Drug Metab. Dispos. 37, 2145-2152 (2009).

6. Tang, M. et al. Antiplatelet agents aspirin and clopidogrel are hydrolyzed by distinct carboxylesterases, and clopidogrel is transesterificated in the presence of ethyl alcohol. J. Pharmacol. Exp. Ther. 319, 1467-1476 (2006).

7. Zhu, H.J., Wang, X., Gawronski, B.E., Brinda, B.J., Angiolillo, D.J. \& Markowitz, J.S. Carboxylesterase 1 as a determinant of clopidogrel metabolism and activation. J. Pharmacol. Exp. Ther. 344, 665-672 (2013).

8. Bouman, H.J. et al. Paraoxonase-1 is a major determinant of clopidogrel efficacy. Nat. Med. 17, 110-116 (2011).

9. Bates, E.R., Lau, W.C. \& Angiolillo, D.J. Clopidogrel-drug interactions. J. Am. Coll. Cardiol. 57, 1251-1263 (2011).

10. Olesen, J.B. et al. Calcium-channel blockers do not alter the clinical efficacy of clopidogrel after myocardial infarction: a nationwide cohort study. J. Am. Coll. Cardiol. 57, 409-417 (2011).

11. Lewis, J.P. et al. The functional G143E variant of carboxylesterase 1 is associated with increased clopidogrel active metabolite levels and greater clopidogrel response. Pharmacogenet. Genomics 23, 1-8 (2013).

12. Zhu, H.J., Appel, D.I., Johnson, J.A., Chavin, K.D. \& Markowitz, J.S. Role of carboxylesterase 1 and impact of natural genetic variants on the hydrolysis of trandolapril. Biochem. Pharmacol.77, 1266-1272 (2009).

13. Thomsen, R., Rasmussen, H.B. \& Linnet, K.; INDICES Consortium. In vitro drug metabolism by human carboxylesterase 1 : focus on angiotensin-converting enzyme inhibitors. Drug Metab. Dispos. 42, 126-133 (2014).

14. Satoh, T. \& Hosokawa, M. Structure, function and regulation of carboxylesterases. Chem. Biol. Interact. 162, 195-211 (2006).

15. Laizure, S.C., Herring, V., Hu, Z., Witbrodt, K. \& Parker, R.B. The role of human carboxylesterases in drug metabolism: have we overlooked their importance? Pharmacotherapy 33, 210-222 (2013).

16. Drummer, O.H., Nicolaci, J. \& lakovidis, D. Biliary excretion and conjugation of diacid angiotensin-converting enzyme inhibitors. J. Pharmacol. Exp. Ther. 252, 1202-1206 (1990).

17. Pang, K.S., Barker, F. 3rd, Cherry, W.F. \& Goresky, C.A. Esterases for enalapril hydrolysis are concentrated in the perihepatic venous region of the rat liver.J. Pharmacol.Exp. Ther. 257, 294-301 (1991).

18. Vistoli, G., Pedretti, A., Mazzolari, A. \& Testa, B. In silico prediction of human carboxylesterase-1 (hCES1) metabolism combining docking analyses and MD simulations. Bioorg. Med. Chem. 18, 320-329 (2010).

19. Vertes, V. \& Haynie, R. Comparative pharmacokinetics of captopril, enalapril, and quinapril. Am. J. Cardiol. 69, 8C-16C (1992).

20. Kuliczkowski, W. et al. Interindividual variability in the response to oral antiplatelet drugs: a position paper of the Working Group on antiplatelet drugs resistance appointed by the Section of Cardiovascular Interventions of the Polish Cardiac Society, endorsed by the Working Group on Thrombosis of the European Society of Cardiology. Eur. Heart J. 30, 426-435 (2009).

21. Sibbing, D., Byrne, R.A., Bernlochner, I. \& Kastrati, A. High platelet reactivity and clinical outcome - fact and fiction. Thromb. Haemost. 106, 191-202 (2011).

22. Shuldiner, A.R. et al. Association of cytochrome $\mathrm{P} 4502 \mathrm{C} 19$ genotype with the antiplatelet effect and clinical efficacy of clopidogrel therapy. JAMA 302, 849-857 (2009).

23. Frelinger, A.L. 3rd et al. Clopidogrel pharmacokinetics and pharmacodynamics vary widely despite exclusion or control of polymorphisms (CYP2C19, ABCB1, PON1), noncompliance, diet, smoking, co-medications (including proton pump inhibitors), and pre-existent variability in platelet function. J. Am. Coll. Cardiol. 61, 872-879 (2013).

24. Angiolillo, D.J. et al. Impact of platelet reactivity on cardiovascular outcomes in patients with type 2 diabetes mellitus and coronary artery disease. J. Am. Coll. Cardiol. 50, 1541-1547 (2007).

25. Angiolillo, D.J. et al. Randomized comparison of a high clopidogrel maintenance dose in patients with diabetes mellitus and coronary artery disease: results of the Optimizing Antiplatelet Therapy in Diabetes Mellitus (OPTIMUS) study. Circulation 115, 708-716 (2007). 
26. Hosokawa, M. et al. Genomic structure and transcriptional regulation of the rat, mouse, and human carboxylesterase genes. Drug Metab. Rev. 39, 1-15 (2007).

27. Marsh, S. et al. Pharmacogenomic assessment of carboxylesterases 1 and 2. Genomics 84, 661-668 (2004).

28. Zhu, H.J. et al. Two CES1 gene mutations lead to dysfunctional carboxylesterase 1 activity in man: clinical significance and molecular basis. Am. J. Hum. Genet. 82, 1241-1248 (2008).

29. Fukami, T., Takahashi, S., Nakagawa, N., Maruichi, T., Nakajima, M. \& Yokoi, T. In vitro evaluation of inhibitory effects of antidiabetic and antihyperlipidemic drugs on human carboxylesterase activities. Drug Metab. Dispos. $\mathbf{3 8}$, 2173-2178 (2010).

30. Takahashi, S., Katoh, M., Saitoh, T., Nakajima, M. \& Yokoi, T. Different inhibitory effects in rat and human carboxylesterases. Drug Metab. Dispos. 37, 956-961 (2009).

31. Shi, D. et al. Anti-influenza prodrug oseltamivir is activated by carboxylesterase human carboxylesterase 1, and the activation is inhibited by antiplatelet agent clopidogrel. J. Pharmacol. Exp. Ther. 319, 1477-1484 (2006).

32. Zhu, H.J., Appel, D.I., Peterson, Y.K., Wang, Z. \& Markowitz, J.S. Identification of selected therapeutic agents as inhibitors of carboxylesterase 1: potential sources of metabolic drug interactions. Toxicology 270, 59-65 (2010).

33. Floyd, C.N., Passacquale, G. \& Ferro, A. Comparative pharmacokinetics and pharmacodynamics of platelet adenosine diphosphate receptor antagonists and their clinical implications. Clin. Pharmacokinet. 51, 429-442 (2012).

34. Wiviott, S.D. et al.; TRITON-TIMI 38 Investigators. Prasugrel versus clopidogrel in patients with acute coronary syndromes. N. Engl. J. Med. 357, 2001-2015 (2007).

35. Cannon, C.P. et al.; PLATelet inhibition and patient Outcomes Investigators. Comparison of ticagrelor with clopidogrel in patients with a planned invasive strategy for acute coronary syndromes (PLATO): a randomised double-blind study. Lancet 375, 283-293 (2010).

36. Takahashi, M., Pang, H., Kawabata, K., Farid, N.A. \& Kurihara, A. Quantitative determination of clopidogrel active metabolite in human plasma by LC-MS/ MS. J. Pharm. Biomed. Anal. 48, 1219-1224 (2008).
37. Tuffal, G. et al. An improved method for specific and quantitative determination of the clopidogrel active metabolite isomers in human plasma. Thromb. Haemost. 105, 696-705 (2011).

38. Andersen, T.F., Madsen, M., Jørgensen, J., Mellemkjoer, L. \& Olsen, J.H. The Danish National Hospital Register. A valuable source of data for modern health sciences. Dan. Med. Bull. 46, 263-268 (1999).

39. Gaist, D., Sørensen, H.T. \& Hallas, J. The Danish prescription registries. Dan. Med. Bull. 44, 445-448 (1997).

40. Sørensen, R. et al. Initiation and persistence with clopidogrel treatment after acute myocardial infarction: a nationwide study. Br.J. Clin. Pharmacol.66, 875-884 (2008).

41. Lamberts, M. et al. Bleeding after initiation of multiple antithrombotic drugs, including triple therapy, in atrial fibrillation patients following myocardial infarction and coronary intervention: a nationwide cohort study. Circulation 126, 1185-1193 (2012).

42. Cunningham, A., Stein, C.M., Chung, C.P., Daugherty, J.R., Smalley, W.E. $\&$ Ray, W.A. An automated database case definition for serious bleeding related to oral anticoagulant use. Pharmacoepidemiol. Drug Saf. 20, 560-566 (2011).

43. Madsen, M., Davidsen, M., Rasmussen, S., Abildstrom, S.Z. \& Osler, M. The validity of the diagnosis of acute myocardial infarction in routine statistics: a comparison of mortality and hospital discharge data with the Danish MONICA registry. J. Clin. Epidemiol. 56, 124-130 (2003).

44. Krarup, L.H., Boysen, G., Janjua, H., Prescott, E. \& Truelsen, T. Validity of stroke diagnoses in a National Register of Patients. Neuroepidemiology 28, 150-154 (2007).

45. Tu, J.V., Austin, P.C., Walld, R., Roos, L., Agras, J. \& McDonald, K.M. Development and validation of the Ontario acute myocardial infarction mortality prediction rules. J. Am. Coll. Cardiol. 37, 992-997 (2001).

46. Rasmussen, S., Zwisler, A.D., Abildstrom, S.Z., Madsen, J.K. \& Madsen, M. Hospital variation in mortality after first acute myocardial infarction in Denmark from 1995 to 2002: lower short-term and 1-year mortality in highvolume and specialized hospitals. Med. Care 43, 970-978 (2005). 